\title{
LA INTERACCIÓN \\ PROFESORADO-ALUMNADO \\ EN LAS CLASES UNIVERSITARIAS, \\ INFLUENCIA DEL CLIMA DEL AULA
}

\section{The teacher-student interaction in the university stage: the influence in the classroom atmosphere}

\author{
Antonio Palomino Martín \\ Correo-e: tpalomino@gmail.com \\ Eduardo Ramos Verde \\ Universidad de las Palmas de Gran Canaria \\ Correo-e: eduardo.ramos@ulpgc.es
}

Recibido: I8 de agosto de 2018

Envío a informantes: is de septiembre de 2018

Aceptación definitiva: I2 de julio de 2019

Resumen: Con este trabajo estudiamos la interacción que se produce en algunas aulas universitarias entre el profesorado y el alumnado en las distintas ramas de conocimiento. Participaron 15 profesores y 560 alumnos. Los resultados nos permiten afirmar que hay diferencias considerables entre las categorías de Distancia y Cercanía, mostrando la primera un total de 359 evidencias por 532 de la segunda respectivamente, lo que indica una clara inclinación hacia una interacción positiva entre el profesorado y el alumnado.

Palabras Clave: interacción profesorado-alumnado; aula universitaria; clima del aula.

AвsтRACт: In this study, we analyze the interaction that occurs in some university classrooms between teacher and student in the various branches of knowledge. They involved is teachers and 560 students. The analyzes allow us to say that there are very significant differences between categories of Distance and Closeness, showing the first of a total of 359 evidence against the 532 of the second, indicating a clear inclination towards positive interaction between teachers and students.

KEY WORDS: teacher-student interaction's; university classroom; distance and closeness. 


\section{Introducción}

$\mathrm{L}$

A TRANSFORMACIÓN DE LA UNIVERSIDAD ESPAÑola en los últimos años ha sido evidente, como consecuencia del conocido «Proceso de Bolonia» o «Convergencia Europea», dentro del Espacio Europeo de Educación Superior. En este ámbito se han estudiado diferentes perspectivas, como los títulos académicos y su influencia en el mercado laboral; qué contenidos hay que impartir; las competencias de estos; la reducción de años, etc. Sin embargo, poco se ha discutido sobre la calidad que se está obligado a impartir en este nuevo escenario y en estas nuevas condiciones.

La calidad educativa en la etapa universitaria es siempre un objetivo que implica o debe implicar a todos los sectores de la sociedad.

La búsqueda de dicha calidad implica una revisión integral de la universidad que incluye estudios sobre el rendimiento académico del alumnado, por lo que sus resultados son un insumo importante, pues permiten conocer elementos obstaculizadores y facilitadores, en consecuencia, permiten favorecer el control de los recursos estatales y la mediación del impacto social (Garbanzo, 2007).

La calidad educativa, considerada como un elemento esencial en el proceso de enseñanza-aprendizaje, no recibe, sin embargo, la atención adecuada por parte de las instituciones, toda vez que no dedican excesivo esfuerzo a la actualización didáctica. Si bien existe gran preocupación por cumplir los planes docentes, por asignar profesorado a cada materia, por obtener unos óptimos resultados del aprendizaje, incluso una tendencia a cumplir la tasa de éxito que se asignó en la elaboración de los Grados, no encontramos la misma preocupación en aspectos como la elección del docente más adecuado para la formación en una materia determinada, en las metodologías empleadas o, lo que es lo mismo, el modo en que se están impartiendo los conocimientos de cada una de las materias; no obstante, la universidad forma profesionales para integrarlos en la sociedad.

A tenor de lo que se desprende de los distintos estudios sobre las modalidades didácticas predominantes en nuestras aulas, en las que muchos profesores actúan como verdaderos «bustos parlantes», muchos de ellos podrían ser reemplazados ventajosamente si se considera el costo de un profesor en relación al de un programa multimedia con escasa capacidad interactiva (Monereo y Pozo, 2003).

La multitud de títulos y por tanto la variedad de contenidos a impartir en la universidad española, con las metodologías condicionadas a estos, nos lleva a sugerir que un docente competente se ajusta al contexto en que enseña, es decir, a la materia que imparte primeramente, también a los requerimientos institucionales (que cada vez son mayores), pero sobre todo a las características de sus alumnos, con una evolución muy rápida y que despiertan no pocas quejas del profesorado; quizás excesivas, por la brecha temporal entre docente y discente. Para Martín (2007) los alumnos valoran las normas a las que deben sentirse sujetos, la afiliación y la implicación en su propio proceso de formación, pero es obvio que esto no evita los condicionantes de la interacción entre ambos.

Fernández (1989) vincula el proceso de socialización (actuación en el aula) del profesor universitario, en buena medida, a sus experiencias como alumno, en especial a 
los modelos docentes que le impactaron, a la presión de la estructura organizativa de su departamento y a las expectativas de sus propios alumnos en cuanto al nivel de exigencia y a las relaciones de la materia con el ejercicio profesional.

Otro debate como la investigación universitaria y su influencia sobre la calidad del profesorado mantiene mitos tan arraigados como el de considerar que los profesores que investigan son mejores docentes porque ofrecen a sus alumnos una perspectiva más viva y actualizada de la disciplina (Monereo y Pozo, 2003). En un análisis realizado en cincuenta y ocho trabajos de investigaciones en las que se estudiaba la relación entre investigación (medida por la cantidad y calidad de publicaciones) y docencia (medida por la calificación hecha por los estudiantes) en departamentos de Ciencias Naturales, Ciencias Sociales y Humanidades, se halla una correlación muy baja entre ambos factores (Hattie y Clinton, citados en Briones, 200I).

Todo ello nos debe hacer pensar que el concepto de calidad para el profesorado universitario, y de alguna forma para el profesorado en general, está condicionado por demasiados factores: desde el tipo de contenidos a impartir, las metodologías más apropiadas, el indiscriminado reparto de la carga docente, las instalaciones, los recursos, hasta los procesos administrativos a los que nos encontramos sometidos.

La función docente del profesorado universitario y los procesos de aprendizaje del alumnado muchas veces están lejos de coincidir, a menudo se oponen. Uno de los malestares del profesorado es la relación entre cuidar y servir en el desempeño docente (Mora y Pujal i Llombart, 20I7).

Como todo proceso educativo, se hace necesario un estudio a fondo de sus condiciones; en nuestro caso, el aula entendida como un proceso social donde interactúan dos agentes, el profesorado y el alumnado.

Saber las características del conocimiento que se transmite en las clases, qué tipo de tareas, el quehacer de alumnos y profesores en el aula nos permite disponer de herramientas para conocer qué formación adquieren los alumnos en su paso por la institución escolar y qué podría hacerse para mejorarla (Merchán, 2009).

El aula como caja negra de la educación no se refiere solo a lo que en ella ocurre, sino también a la dificultad que tiene el desciframiento de sus claves; el desarrollo de la clase, que constituye un escollo en la investigación práctica, y los agentes presentes, alumnos y profesores, informantes imprescindibles (Depaepe, 2006). Lo que se produce en las aulas universitarias es importante para conocer si la formación que impartimos está en las mejores condiciones y si las interacciones en dichas aulas propician la enseñanza, pero sobre todo el aprendizaje.

Para Rego (2017) la formación pedagógica del profesorado en el futuro de la universidad es un vector de cambio y de innovación sostenida, en el marco de mejora de la reputación de las universidades. Las conclusiones de sus trabajos advierten sobre posibles reduccionismos instrumentales en la formación pedagógica, en un mundo global, el aprendizaje supone capacidades vinculadas a la resolución de problemas y el diseño de proyectos en nuevos entornos.

\section{El clima del aula}

El estudio del aula requiere del conocimiento de lo particular; se hace imprescindible su contexto para ser investigado. Autores como Yin (1989) destacan la contextualización al entender que un estudio de casos es una investigación empírica dirigida 
a un fenómeno contemporáneo dentro de su contexto real por la imposibilidad de separar las variables de estudio de su contexto. Para Walker (1983), «es el examen de un ejemplo en acción».

Si bien, el problema con el que estamos tratando es que apenas existen trabajos que documenten el clima escolar en las aulas universitarias, la gran mayoría se realizan en la etapa educativa de Secundaria (Carbonero, Martín-Antón y Reoyo, 20II).

La expresión clima de aula es compleja, es un constructo multidimensional; una parte material que abarca las infraestructuras y otra inmaterial, el tipo de interacción que construyen en la clase, destacando la interacción entre el profesorado y los estudiantes y de los estudiantes entre sí (Milicic y Aarón, 2004).

Para entender mejor este concepto de clima escolar, Coronel (1994) lo caracteriza como un concepto globalizador, multidimensional, donde las características humanas son relevantes, relativamente permanentes, que influye en el logro de los objetivos académicos y desarrollo personal y donde la percepción de las personas constituye un indicador fundamental del estudio del clima.

Biggs (2005) define el clima de aula como «la forma en que los estudiantes y los académicos tienden a sentir las cosas, producto de sus interacciones sociales, formales e informales, lo que tendría efectos sobre el aprendizaje de los primeros».

Todo lo que envuelve un aula condiciona la interacción entre profesorado-alumnado, tanto las expectativas del profesorado como la motivación del alumnado. La falta de motivación en las aulas se ha convertido en uno de los problemas centrales de la educación en España (Valle, Cabanach y Rodríguez, 2006). Para Alonso-Tapia (2005) existen varios estudios que muestran cómo la motivación influye significativamente en el aprendizaje del alumnado.

El orden en la enseñanza se interpreta por unas determinadas pautas de comportamiento de los alumnos: silencio, atención, obediencia, etc. Por diversos motivos, los estudiantes tienden a producir alteraciones de manera significativa; por su parte, los profesores tratan de producir el orden que consideran necesario. En este sentido la vida en el aula es estructuralmente conflictiva, pues buena parte de los objetivos que persiguen alumnos y profesores son contradictorios. Las relaciones y la producción de un aula no pueden estar supeditadas a los contenidos que se imparten, a la metodología más apropiada o las tareas que se desarrollan, se hace necesaria la formulación de una teoría de la acción en el aula, la enseñanza, más que una práctica de transmisión y adquisición de conocimiento en el aula, es una práctica social que opera en un contexto concreto (Merchán, 2005).

El análisis de la actuación de los alumnos tiene que ser reformulado, más allá de una perspectiva cognitiva, el modo de proceder de estos es decisivo en la configuración de la enseñanza. En este sentido, se hace necesario cuestionar la idea de que el alumno sea fundamentalmente un aprendiz, alguien que acude a clase con el propósito de adquirir conocimiento y que actúa con arreglo a esa condición. Existen distintos tipos de alumnos, distintos modos de afrontar su presencia en el aula y su relación con lo que la institución educativa proporciona y distintos modos de intervenir (Merchán, 2005).

Para Gairín (1999) las instituciones educativas pueden considerarse organizaciones sociales, conformadas por un grupo de personas que ejercen determinadas funciones, dirigidas hacia la búsqueda de la eficiencia y la racionalidad. Así, Molina de Colmenares y Pérez de Maldonado (2006) defienden que, a la cultura de las organizaciones, 
se le vincula el concepto de clima, definido por diferentes autores en el área de la gerencia y de la administración.

En todos los contextos educativos, se produce un proceso recíproco donde las personas que se ponen en contacto valoran los comportamientos de los otros y se forman opiniones acerca de ellos, todo lo cual suscita sentimientos que influyen en el tipo de relaciones que se establecen (Saballs y Castañer, 2002).

La carencia de habilidad para enseñar, así como las estrategias a usar, contribuye a la desmotivación del alumnado, a su poca implicación en las tareas y, por tanto, al bajo rendimiento académico y deterioro del clima escolar (Fernández, 2008; Benito, 2009 y Carrasco, 2010).

El clima de aula se dificulta en la universidad, debido a unas relaciones profesorado-alumnado condicionadas por un sistema que adquiere formas que antes eran desconocidas. Para Valle Olmos (2013) en un trabajo que aborda el deterioro de la relación entre profesores y alumnos en la universidad y con la intención de contribuir a la mejora de un problema social que existía en la Educación Secundaria y que ha empezado a manifestarse en el ámbito universitario, se describen una serie de factores que influyen en dicho clima, como el tipo de asignatura, clases masificadas, presión del alumnado con envío masivo de e-mails, profesores que ven al alumno como «enemigo", alumnos que se sienten clientes y creen tener la razón, profesores en continua alerta por las reclamaciones a estancias superiores. También describe las relaciones desde el alumnado como falto de disciplina, inmadurez, todo vale, pérdida de atención con las nuevas tecnologías, hablan mucho, ambiente propio de un instituto, etc. Muchas de estas causas son producidas por la implantación de los Grados, que hacen obligatoria la asistencia y, por tanto, conlleva que un porcentaje muy elevado del alumnado, poco motivado, provoque desorden en las aulas, deteriorando el clima del aula y, por tanto, la interacción del profesorado-alumnado.

Se extiende la opinión entre profesores y alumnos de que en las aulas hay menos disciplina que antes y que «todo vale».

Algunos autores enfatizan el clima de aula universitario desde el análisis de los contenidos; otros señalan la cultura y la construcción de conocimientos, y hay quienes optan por destacar el aporte de las emociones, la comunicación, las relaciones interpersonales, las relaciones de poder en el aula, así como las características del clima de aula que en ellas se desarrolla (Pereira-Pérez, 20ıo).

Un estudio hecho por Hernández (2005) con docentes y estudiantes universitarios coincide en que los factores afectivos son de suma importancia para un adecuado clima de aula y para lograr mejores aprendizajes. Además, ambos concluyen que este es un tema poco tratado en el contexto universitario.

Dicho clima se favorece cuando el docente universitario asume el rol de «facilitador del aprendizaje» y, también, cuando se interesa por la formación integral de sus estudiantes (Hannan y Silver, 2005). Está sujeto a muchos condicionantes, pero casi todos los autores coinciden que su mejora redunda en que el docente tenga dominio del contenido, metodología motivadora y variada, una evaluación justa que mida aprendizajes profundos y una interacción de respeto con los estudiantes (Milicic y Aarón, 2004; Biggs, 2005 y Perrenoud, 2005). Además, deben primar las relaciones centradas en el entendimiento, la comprensión, los intereses y las motivaciones de los diferentes actores, haciendo predominar un clima de cordialidad, respeto y confianza. Es importante la voluntad del docente en dirigir las acciones para crear dicho ambiente (Ríos, 20ıo). 
Los actuales enfoques en la investigación de los procesos de aula describen las clases como realidades complejas. Sus componentes no son visibles y hacen difícil incorporar una escala de observación. Los métodos han de ser multidimensionales y holísticos. Se puede tener una «visión» de la enseñanza tomando tanto el contexto como la interacción social entre los agentes y el intercambio comunicativo entre ellos (Sabucedo, 200I).

\section{La interacción profesor-alumno en la etapa universitaria}

Cuando un grupo de alumnos y el profesorado desarrollan conjuntamente una actividad, necesariamente estamos obligados a detenernos en qué ocurre. Sin duda, esta interacción es uno de los elementos que más condiciona el ambiente o el clima de un aula. Podemos referirla (Escudero, 1997) como el conjunto de transacciones sobre determinados contenidos. Estos, a su vez, podrán referirse, por un lado, a cuestiones cognitivas, instructivas, objetivas, al servicio de la información. Se evidenciaría este tipo de situación cuando el profesor interactúa con los estudiantes para explicarles una cuestión propia de la materia o para darles instrucciones sobre cómo hay que proceder en una actividad. Pero la interacción también surge, por otro lado, en torno a cuestiones afectivas. Cuando le decimos a un alumno «me gusta lo que hiciste» o le dirigimos una mirada intensa ante un mal comportamiento, estamos generando una transacción comunicativa a partir de cuestiones afectivas. Es evidente que dicha interacción también puede producirse de manera simultánea a la comunicación de cuestiones cognitivas.

Una interacción positiva ha sido igualmente subrayada como factor clave cuando se desarrollan prácticas de aula inclusivas que aceptan las diferencias del alumnado (Ainscow y Tomlison, 200I). En este sentido, cobra interés lo planteado por Voli (2004), cuando afirma que el modelo de persona que el profesor proporciona a sus estudiantes contribuye a la formación de la personalidad de estos, por lo cual el educador debe asumir con gran responsabilidad la creación de un ambiente favorecedor de la convivencia en el aula. La relación estrecha entre interacción y mejora de la convivencia ha sido también analizada en trabajos anteriores (Marchena, 2007).

El «buen profesor» según los alumnos (Bahillo, Puyal, Sanz, Elboj y San Agustín, 2006) se define atribuyéndole singularidades profesionales (preparación de clases, los contenidos que explica son interesantes...), acompañadas siempre de otras sociales: se puede establecer una relación con él, es afable, tiene sentido del humor, se preocupa por nosotros, jamás descalifica, no se burla de nadie, no es sarcástico. Estas relaciones son opuestas cuando los estudiantes quieren, por el contrario, definir al «mal profesor».

Desde el punto de vista del alumnado, la interacción que se establece con el profesor es el componente al que se le concede mayor trascendencia. Un trabajo realizado con mil estudiantes universitarios (Ibáñez, 2002) sobre la percepción de su escolaridad básica concluyó que la calidad de sus relaciones con los profesores fue lo que mayor impacto tuvo entre ellos, y no tanto el contenido de estudio.

Nuestro trabajo se centra en un paradigma interpretativo/ecológico y ha sido desarrollado bajo un enfoque etnográfico y cualitativo. En él se analiza la interacción que mantiene el profesorado con su alumnado a partir de la observación directa de lo que ocurre en algunas clases universitarias de las distintas ramas de conocimiento. 
Tal como afirman Echeverri y López (2005), el currículo es un sistema complejo no solamente porque comporta variados componentes (sujetos, saberes, intencionalidades, ambientes, didácticas y evaluación), sino sobre todo porque mantiene operaciones e interacciones por medio de las cuales se derivan nuevos sistemas. Cada uno de estos nuevos sistemas tiene su propia organización, pero requiere de la interacción con los otros, en la medida en que estos le permiten flujos de información. En este sentido, la tesis de considerar al aula meramente como un campo de reproducción está rebatida con argumentos y datos muy solventes. A este respecto, en sus trabajos sobre la historia del currículo, Goodson (2000) plantea la idea de que este se construye en diversos ámbitos y niveles, distinguiendo entre el «currículo escrito» y el "currículo como actividad en el aula», y llama la atención sobre el peligro de estudiar únicamente el currículo que se explicita en los programas de estudio, en las prescripciones oficiales o en los libros de texto, olvidando que «las altas esferas del currículo escrito están sujetas a renegociación a niveles más bajos, y especialmente en el aula» (Goodson, 1991: 30). Según esto, podríamos decir que el currículo se construye en diversos campos, «ámbitos» o «niveles», de entre los que cabe destacar el campo del currículo oficial, el campo de los libros de texto y el campo de la práctica. Por nuestra parte, como docentes, no podemos seguir pensando que estar motivado no es una condición individual del alumno, algo ajeno y previo a nuestra práctica, sino que cambiar esos motivos debe ser también una de las metas que guíe la práctica docente, generar nuevas metas de aprendizaje debe ser uno de los efectos colaterales de la puesta en «marcha de nuevas prácticas docentes», como puede verse, por ejemplo, en los trabajos de Cabaní y Carretero (2003). Para que las distintas competencias incidan en los estudiantes, resulta indispensable que se promuevan cambios radicales en los otros dos factores: el currículo que se imparte, sobre el que reflexiona ampliamente Coll (2003), y los profesores que lo imparten, cuyo retrato nos acerca Gairín (2003).

Por todo lo expuesto, en este estudio se analizaron las interacciones en el aula entre el profesorado y el alumnado en diferentes grados universitarios de cada una de las cinco ramas de conocimiento. Una vez analizadas las evidencias de los registros narrativos obtenidos en cada una de las clases registradas, se establecieron las diferencias entre aquellas categorías que pertenecen a la variable de Distancia y la variable Cercanía.

\section{Método}

El método aplicado fue etnográfico, acogiéndonos a un estudio de casos colectivos como estrategia de diseño (Stake, 2005), de corte observacional (Heras-Montoya, 1997). Se siguió un paradigma ecológico e interpretativo de investigación en el aula (Doyle, 1979; Tikunoff, Ward y Griffin, 1979 y Pérez Serrano, 1994).

\section{Muestra}

Se procedió a la selección siguiendo un muestreo intencional, se tomaron cada una de las cinco ramas del conocimiento: Artes y Humanidades; Ciencias Sociales y Jurídicas; Ciencias de la Salud; Ingeniería y Arquitectura; Ciencias (Real Decreto 
1393/2007, de 29 de octubre). Se eligieron quince profesores de diferentes grados, tres por cada rama del conocimiento, todos ellos con la categoría de doctor en cada una de las asignaturas impartidas.

Sumados los grupos de clases, oficialmente en listas de matriculación de los profesores, se contabilizaron un total de 560 alumnos, lo que se traduce en 37.3 alumnos por media de clase estudiados.

En la recogida de datos se emplearon un total de 75 sesiones de clase (con un promedio de 50 minutos), de las que 6o fueron analizadas; se asignaron cinco sesiones de estudio para cada profesor, pero solo cuatro fueron analizadas para dejar la primera de familiarización con nuestra presencia. Todas las clases fueron en el aula, descartando las sesiones prácticas para unificar criterios en la recogida de datos. El número de sesiones se determinó adoptando los acuerdos de Glaser y Strauss (1967: 6I-62) y su descripción de la «saturación teórica», planteando que, en los estudios de casos, muchas veces el investigador intenta recabar la mayor información posible, sin embargo, llega un momento en el que por más que el observador siga recogiendo datos obtendrá las mismas conclusiones.

Durante el trabajo de recogida de evidencias (entendidas como los hechos que se producen en la interacción del aula, siendo destacables para registrar y posteriormente analizar) el observador se mantuvo siempre al margen, intentando no influir en el desarrollo de la sesión, situado en una posición que le permitiera observar todo lo ocurrido. Se desarrolló también una observación persistente (Del Villar, 1994) para comprender mejor nuestro objeto de estudio y evitar la precipitación de los juicios.

\section{Instrumentos de medida}

Para extraer la información empleamos un sistema narrativo, uno de los muchos sistemas existentes para registrar y almacenar información (Evertson y Green, I989). Este sistema se concretó elaborando múltiples notas de campo dando lugar a un relato por escrito de lo que el investigador ve, oye, experimenta y piensa durante su actividad de recoger datos. Posteriormente y para poder extraer las evidencias (hechos acontecidos) de estos registros narrativos, garantizando la validez interna y la fiabilidad del estudio, se realizó una triangulación metodológica de investigadores (Stake, 2005).

El sistema categorial que se elaboró para clasificar los registros narrativos obtenidos en las sesiones en la fase analítica de la investigación tuvo carácter tanto inductivo como deductivo, negociándose los significados de manera conjunta con todo el equipo investigador. En la Tabla i mostramos las categorías y subcategorías resultantes junto con su significado. El referente teórico para las categorías extraídas de manera deductiva se basó en un trabajo anterior donde se analizaron los mismos elementos, pero con otras materias curriculares (Marchena, 2005). Posteriormente fueron modificadas y adaptadas por Marchena, Palomino, Ramos y Montesdeoca (20I7). Para facilitar el manejo de la información cualitativa, se hallaron las frecuencias y porcentajes surgidos en esta fase del análisis. 
LA INTERACCIÓN PROFESORADO-ALUMNADO EN LAS CLASES UNIVERSITARIAS, INFLUENCIA DEL CLIMA DEL AULA ANTONIO PALOMINO MARTÍN Y EDUARDO RAMOS VERDE

TABLA I: Categorización de «Interacción profesorado-alumnado»

\begin{tabular}{|c|c|}
\hline \multicolumn{2}{|r|}{ INTERACCIÓN PROFESORADO-ALUMNADO } \\
\hline \multirow{7}{*}{$\begin{array}{c}\text { Distancia: } \\
\text { Toda la comunicación } \\
\text { que es considerada } \\
\text { desfavorable en } \\
\text { la interacción } \\
\text { profesorado-alumnado }\end{array}$} & $\begin{array}{l}\text { ANTAGONISMO: se producen situaciones de disconformidad y/o } \\
\text { castigo. }\end{array}$ \\
\hline & $\begin{array}{l}\text { TENSIÓN ENCUBIERTA: se producen mensajes secos, indirectos, iró- } \\
\text { nicos, tajantes e impactantes. }\end{array}$ \\
\hline & $\begin{array}{l}\text { VELOCIDAD: el profesorado exige o explica tareas con rapidez y el } \\
\text { alumnado se incomoda. }\end{array}$ \\
\hline & OMisión: ausencia de feedback solicitado por el alumnado. \\
\hline & InHIBICIÓN: ausencia de respuesta ante situaciones conflictivas. \\
\hline & $\begin{array}{l}\text { FAvoritismo: se atiende de forma más favorable a un alumnado } \\
\text { que a otro. }\end{array}$ \\
\hline & DESCRÉDITO: se producen mensajes de desvaloración. \\
\hline \multirow{5}{*}{$\begin{array}{c}\text { Cercanía: } \\
\text { Toda la comunicación } \\
\text { que es considerada } \\
\text { favorable en } \\
\text { la interacción } \\
\text { profesorado-alumnado }\end{array}$} & $\begin{array}{l}\text { Personalización: se producen gestos de afecto y/o interés por } \\
\text { cuestiones personales y/o el profesor interactúa como un igual. }\end{array}$ \\
\hline & $\begin{array}{l}\text { HUMOR COMPARTIDO: se producen situaciones de humor entre } \\
\text { ambos. }\end{array}$ \\
\hline & FLEXIBILIDAD: se alcanzan acuerdos comunes con facilidad. \\
\hline & VALORACIÓN: se realizan mensajes de aprobación y felicitación. \\
\hline & Ánimos: se alienta la realización de tareas. \\
\hline
\end{tabular}

Fuente: Elaboración propia.

Se entiende por «interacción profesorado-alumnado» la interacción comunicativa que se desarrolla en las clases, la forma de relacionarse. Siguiendo las teorías sobre liderazgo docente de Fabra y Doménech (200I), el profesorado muestra dos tipos de conductas en el aula, la relativa a las tareas y la que tiene que ver con la relación que mantiene con los estudiantes. Ambas conductas dan lugar a diversos estilos de liderazgo.

\section{Resultados}

Los resultados obtenidos los presentamos separando las interacciones, de forma global (Figuras I y 2) y por ramas de conocimiento (Figuras 3 y 4).

Por una parte, la categoría que denominamos Distancia (Figura I) corresponde a todas las interacciones que consideramos que separan al profesorado del alumnado de forma global y distinguidas por ramas de conocimiento (Figura 3). Por otro lado, las interacciones que consideramos beneficiosas para profesorado y alumnado las denominamos Cercanía (Figura 2) si es global y por ramas de conocimiento la presentamos en la Figura 4.

El total de evidencias obtenidas, en las 60 sesiones analizadas, fue de 89i. En la categoría de Distancia registramos 359, un 40,3\% del total y en Cercanía la cifra ascendió a 532, el 59,7\% de los datos.

Los valores globales de la categoría Distancia (Figura i) están descritos a través de siete subcategorías, con ello pormenorizamos el tipo de distanciamiento que se produce: Antagonismo, Tensión Encubierta, Velocidad, Omisión, Inbibición, Favoritismo 
y Descrédito. Hemos hecho una valoración de las mismas, teniendo en cuenta el número de evidencias obtenidas; el Antagonismo registra 172 evidencias, Tensión Encubierta 173, Omisión 13, Descrédito tan solo una evidencia. El resto de subcategorías, Velocidad, Inbibición y Favoritismo, no presentaron ninguna evidencia.

FiguRA I: Interacción profesorado-alumnado en Distancia

\section{DISTANCIA PROFESORADO-ALUMNADO}

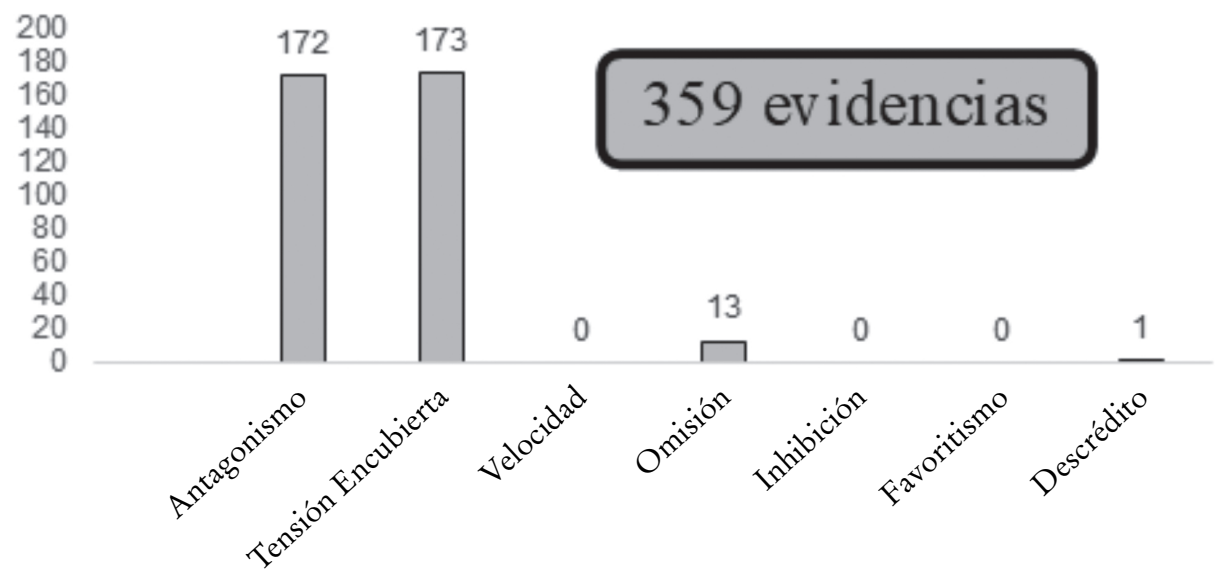

FIGURA 2: Interacción profesorado-alumnado en Cercanía

\section{CERCANÍA PROFESORADO-ALUMNADO}

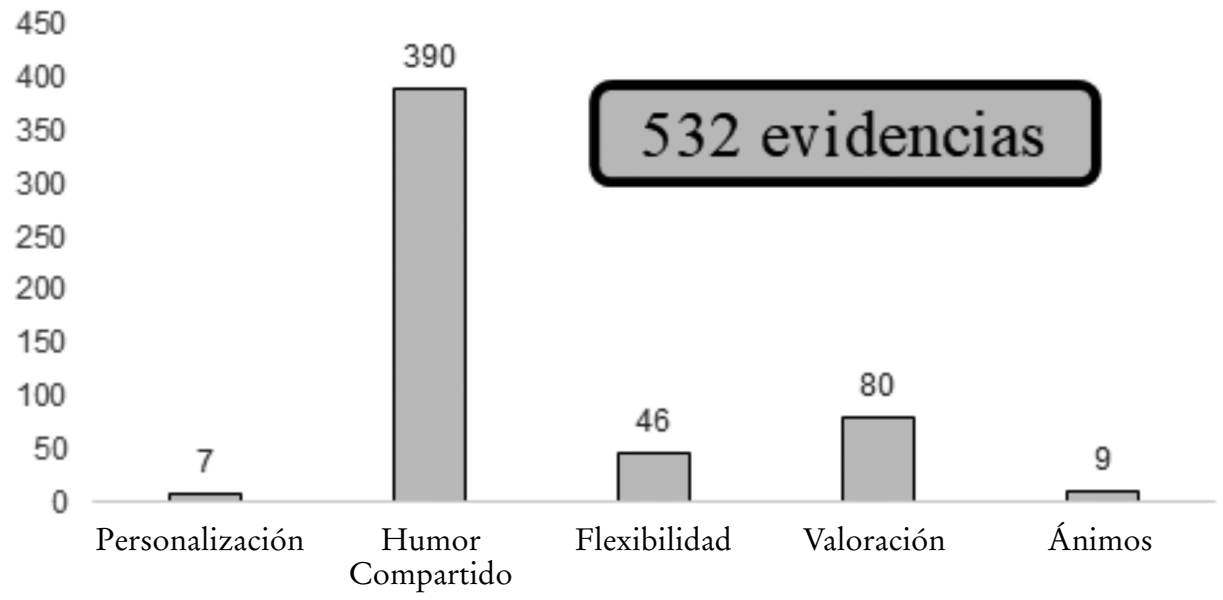

Los valores globales de la categoría Cercanía (Figura 2) están compuestos por cinco subcategorías: Personalización, Humor compartido, Flexibilidad, Valoración y Ánimos. Con un total de 532 evidencias, se produce una gran concentración en la subcategoría de Humor Compartido con 390, seguido de la Valoración con 80, 
Flexibilidad con 46 y finalmente Ánimos y Personalización con 9 y 7 evidencias respectivamente.

Los resultados nos ofrecen otra visión de la interacción si los presentamos por ramas de conocimiento. En la Figura 3 mostramos los valores de Distancia, destacando los tres grados de la rama de Ciencias Sociales y Jurídicas que sobresalen en Antagonismo con II8 evidencias y Tensión Encubierta con I33, junto con las 8 de Omisión, suman un total de 259 y la convierten en la rama con más interacciones en Distancia.

FIGURA 3: Interacción profesorado-alumnado en Distancia por ramas de conocimiento

DISTANCIA PROFESORADO-ALUMNADO

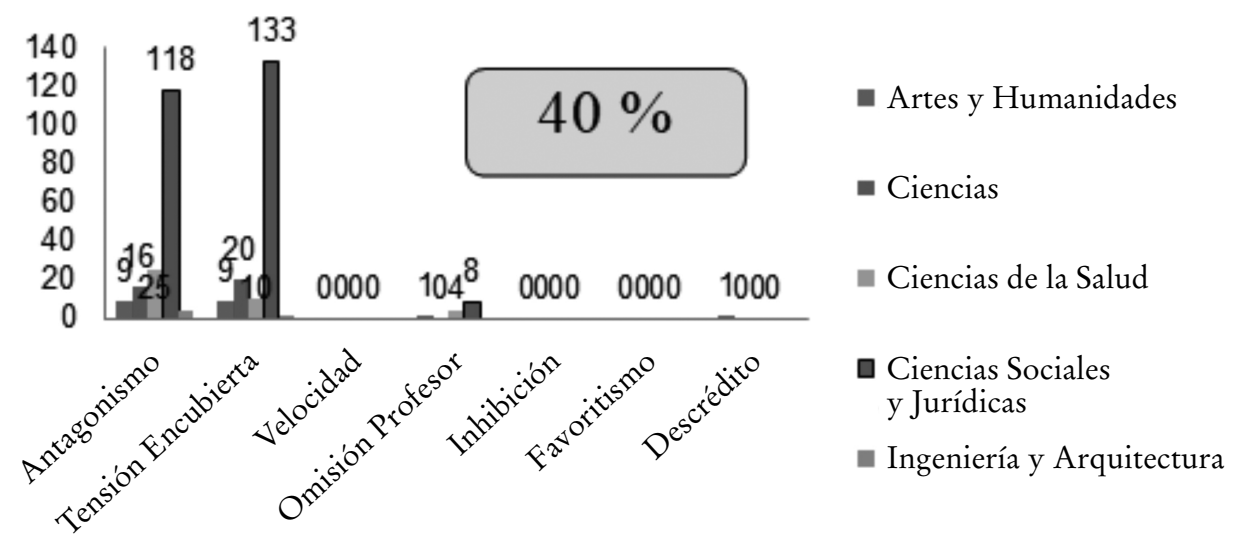

FIGURA 4: Interacción profesorado-alumnado en Cercanía por ramas de conocimiento

\section{CERCANÍA PROFESORADO-ALUMNADO}

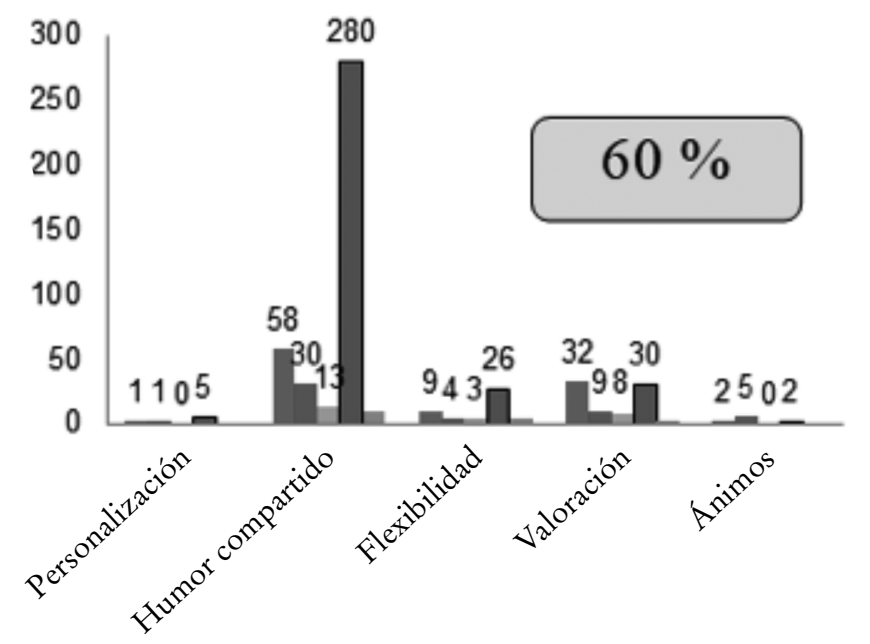

- Cercanía profesoradoalumnado Artes y Humanidades

- Cercanía profesoradoalumnado Ciencias

- Cercanía profesoradoalumnado Ciencias de la Salud

- Cercanía profesoradoalumnado Ciencias Sociales y Jurídicas 
Los nueve grados de las ramas de Arte y Humanidades, Ciencias y Ciencias de la salud presentan evidencias similares. Es la rama de Ingeniería y Arquitectura, con diferencia, quien menos evidencias registró, tan solo cinco.

La Figura 4 presenta los datos de Cercanía por ramas de conocimiento. Destacan también los grados de Ciencias Sociales y Jurídicas con 343 evidencias, principalmente en Humor Compartido con 280 y obteniendo registros en las cinco subcategorías. $\mathrm{Al}$ contrario que la categoría Distancia, la rama de Artes y Humanidades registra, en segundo orden, hasta IO2 evidencias, también es Humor Compartido la subcategoría con mayores evidencias, en este caso 58. Vuelve a ser la rama de Ingeniería y Arquitectura la que menos registra en interacción profesorado-alumnado, tan solo i4 evidencias.

\section{Discusión y conclusiones}

Con los resultados de nuestra muestra, es posible determinar algunas tendencias del proceso de enseñanza-aprendizaje en la etapa universitaria, pero se nos hace difícil responder al debate que la universidad tiene desde hace mucho tiempo sobre la dualidad del profesorado en las funciones docentes e investigadoras. En ningún caso pretendemos entrar en dicho dilema, como ya apuntaron en sus trabajos Monereo y Pozo (2003), de que los profesores designados como mejores investigadores no son los mejores docentes. También en trabajos anteriores como el de Hattie (200I), atribuyendo una puntuación determinada, valoraron a los profesores con el perfil de investigadores y/o docentes, concluyendo que buenos resultados en investigación solían tener bajos resultados en docencia. Conocido este debate y algunos trabajos que apuntan a ciertas tendencias similares, debemos aclarar que nuestro estudio no analiza dicha cuestión, aunque pudieran existir en nuestra muestra docentes de ambas tendencias. Partimos del convencimiento de que el profesorado universitario español realiza las dos funciones con sobrada competencia.

La motivación para investigar la calidad de la enseñanza en la Universidad, en nuestro caso a través del clima del aula como uno de los factores que influyen en ello, coincide con los trabajos de Garbanzo (2007), donde el rendimiento académico es importante para controlar el proceso y poder justificar los cuantiosos recursos que el Estado dedica a esta etapa educativa. Hacer las cosas bien, entre otras, supone mejorar una imagen ante nuestro alumnado y, por tanto, ante la sociedad a la que nos debemos. Todo parte de hacer las cosas bien, de elevar la calidad de nuestra investigación, de nuestra docencia y, para esto, debemos estudiar cómo es la calidad de nuestra enseñanza y el aprendizaje que proporcionamos.

Cuando diferenciamos los registros obtenidos en valores de Distancia y Cercanía, destacan significativamente los que reconocemos como Cercanía en la interacción profesorado-alumnado ( 532 evidencias), es un primer dato que nos indica que el clima del aula es bastante favorable en nuestra universidad, sin diferenciarla, por ahora, en ramas de conocimiento. Es obvio que dicho resultado se suscribe a la muestra de los i6 profesores estudiados y se hace necesaria una muestra mucho más numerosa y a largo plazo para considerar estos datos como una tendencia fiable.

Centrándonos en los resultados que hemos categorizado como Distancia, se produce una interacción alta en antagonismo y tensión encubierta, valores que confirman 
los resultados obtenidos por Valle Olmos (2013) atribuyéndole al aula universitaria la característica de «conflictiva», definiéndola como una continuidad de la Eso. En trabajos anteriores como los de Martín (2007) se obtuvieron conclusiones que atribuyen al alumnado una valoración hacia las normas y, por tanto, un respeto hacia estas. Podemos entender que en los últimos años dichas normas se pueden conocer, pero se respetan menos, como la puntualidad, la atención, el silencio en clase, el respeto del contexto, etc. A través de estas dos subcategorías el profesorado de nuestra muestra ha controlado la tensión producida en el aula y la falta de respeto a los valores citados. La ausencia de respeto a estas formas de convivencia en el aula es un síntoma que perjudica inevitablemente la calidad de nuestra enseñanza y no siempre se produjo por parte del alumnado, aunque sí en la mayoría de los casos. Los trabajos de Merchán (2005) confirman que el aula no transmite solo contenidos y que todo está supeditado a estos, es una práctica social que opera en un contexto concreto, es un espacio social en el que se convive y, por tanto, se interactúa.

En la Distancia de la interacción profesorado-alumnado, la Omisión es la tercera en frecuencia, aunque con un valor bajo (I3 evidencias), son los únicos datos que podemos atribuirles a una mala praxis del profesorado, omitir las preguntas que le hace el alumnado, tanto por desconocimiento o por desinterés, y ambas son acciones que entorpecen este clima.

En nuestro trabajo, dichos casos fueron considerados como falta de habilidad del profesorado, eran los profesores con menos años de docencia, en concreto tres. Coinciden con otras investigaciones como las de Fernández (2008), Benito (2009) y Carrasco (20I0), que lo atribuyen a la falta de habilidad para enseñar, una razón para la desmotivación del alumnado y en consecuencia un aumento de registros en la categoría de Distancia.

Si descartamos el Descrédito, un solo caso y anecdótico, el profesorado universitario no interactúa con Velocidad, Inbibición o Favoritismo, no se dio ni un solo caso en la muestra estudiada. Este resultado contrasta con trabajos similares con profesorado de primaria, secundaria y bachillerato, que aunque no se encuentren en el mismo nivel educativo pueden ser una referencia a comparar en cuanto a clima de aula; Marchena et al. (2017), que con muestras más pequeñas, 9 profesores, en 55 sesiones y con 150 alumnos, estas subcategorías alcanzaron is evidencias de Velocidad, 6 de Favoritismo, 20 Inhibición y 5 Descrédito. Lógicamente son subcategorias que en edades adultas es difícil que se produzcan por lo que representan. Aun así, no es prudente concluir que el profesorado universitario no interactúa en estas variables con sus alumnos, son necesarios estudios posteriores que amplíen la muestra, sobre todo la Velocidad, interacción que siempre se le atribuye al profesorado universitario.

Cuando estudiamos los resultados en la categoría de Cercanía, podemos destacar que en todas las subcategorias se obtuvieron evidencias; aspecto que justifica el elevado número de registros en comparación con la interacción de Distancia.

La subcategoria de Humor Compartido, con 390 evidencias, es la interacción que más se produce en el aula universitaria, con bastante diferencia del resto. Siempre entendiendo que no hablamos de la personalidad del profesor o si este es «gracioso», tan solo que se producen situaciones de humor entre ambos o las clases se dirigen de buen talante o una ironía con humor. Para que se produzcan tantos registros en esta subcategoría es necesario tener un profesorado con cierta predisposición y con una buena motivación. Los trabajos de Voli (2004) atribuyen a la personalidad del profesorado 
cierta influencia, el modelo de persona que se ofrece al alumno contribuye a su formación. Por tanto, una personalidad más cercana, más flexible y con interacciones de mejor talante hace que el profesorado favorezca la Cercanía. Esto lo ratifica Biggs (2005) cuando profesorado y alumnado «sienten» las cosas que se producen en el aula, no son elementos pasivos y, por tanto, tienen efecto sobre el aprendizaje de nuestros alumnos.

Si continuamos en esta línea, la Valoración es la subcategoría en segundo orden, con gran número de evidencias (8o), esto indica que el profesorado estudiado no solo ha transmitido los contenidos que corresponden, es decir, ha generado procesos de enseñanza adecuados, sino que para generar aprendizaje utiliza con frecuencia el recurso de la Valoración de sus alumnos, algo que no tiene que ser extraño en esta etapa educativa. Los trabajos de Saballs y Castañer (2002) determinan que los procesos educativos son de características recíprocas, frecuentemente se opina y siempre se valora lo que hace el otro, aunque en muchas ocasiones no se manifieste. Obviamente, en este caso hablamos de valoración positiva, siempre tenemos una opinión del otro, estemos sentados en uno u otro lado del aula, si la valoración es favorable por parte del profesorado incrementa el parámetro Cercanía.

La Flexibilidad es la tercera más frecuente, aunque pudiera producirse en ambos estamentos, flexibilizar posiciones del profesorado como del alumnado, en nuestro estudio, por el tipo de clases magistrales, las 46 evidencias detectadas provienen del profesorado ante demandas del alumnado, esto conlleva una interacción favorable en la relación y, por tanto, aumento de la afectividad entre ellos. Para Hernández (2005), el factor afectivo es importante, es un elemento favorecedor de un buen clima del aula. Podemos deducir que este tipo de actitudes en clase generan bastante acercamiento con el alumnado.

La subcategoría de Ánimos es una interacción muy representativa de la categoría Cercanía. En nuestra muestra obtuvimos tan solo 9 registros, entendemos que son escasos, incluso para una etapa universitaria. De igual forma, la Personalización, con 7 evidencias, es otra interacción que genera Cercanía y ambas han mostrado pocos registros. Es frecuente que la enseñanza universitaria mantenga una Distancia con el alumnado, por el número de alumnos, tipo de clases, metodologías empleadas, etc. Quizás es buen momento, acompañando los cambios de la convergencia europea, para plantear nuevos modos de trato hacia el alumnado, sobre todo cuando hemos entrado en un contexto en que todos los factores del proceso son evaluables, profesores, alumnos, títulos, procesos, etc.

Si estudiamos los resultados considerando las cinco ramas de conocimiento que determinó el Real Decreto 1393/2007 y sin intención de presentar datos concluyentes, ni universales, es la rama de Ciencias Sociales y Jurídicas la que presenta más evidencias de Distancia (259) y también de Cercania (343), las subcategorias con más evidencias se corresponden con lo analizado de los datos en la totalidad, es decir, Tensión Encubierta (I33) y Humor Compartido (280). Es, por tanto, la rama de conocimiento que aporta más evidencias a la totalidad de nuestro estudio, con diferencia a la segunda rama de conocimiento que es Ciencias de la Salud en Distancia (39) y Artes y Humanidades en Cercanía (IO2). Podemos concluir que los Grados en Derecho, en Ciencias de la Actividad Física y del Deporte y en Profesorado imparten unos contenidos que permiten al profesorado «generar» gran Cercanía con su alumnado, especialmente los dos últimos Grados. Pero también las mayores evidencias en Distancia, desde nuestro punto de vista, por el número tan elevado de alumnos en sus aulas. 
En el lado opuesto, y siguiendo con el análisis por ramas de conocimiento, es Ingeniería y Arquitectura la que presenta menos evidencias en las dos categorías también, tan solo (5) en Distancia y (I4) en Cercanía. Grados con pocos alumnos y sin poder ser concluyentes, se hace necesario estudiar todos sus cursos del Grado para tener datos más objetivos.

La universidad española ha repetido en muchos ámbitos su apuesta por la calidad docente, por tanto, este es un factor que se suma a dicha propuesta. Existen muchos estudios que defienden que un incremento de la calidad puede producirlo si las relaciones entre profesorado y alumnado es propicia. Para Bahillo et al. (2006), al buen profesorado, según los alumnos, se le atribuyen valores profesionales y también sociales. En un trabajo más extenso sobre las relaciones sociales del aula, Ibáñez (2002) concluye que, entre varios factores, son las relaciones con los profesores lo que más impacto tuvo en el alumnado.

De los resultados obtenidos y lo discutido del trabajo, podemos concluir que el profesorado universitario, en general, sin diferenciar la rama de conocimiento a la que pertenece, establece interacciones con su alumnado de bastante cercanía, alejándose de esa figura o idea que teníamos de un profesor distante.

Estudiados por ramas de conocimiento podemos concluir que el contenido que se enseña y la masificación de las aulas condicionan la interacción. Pero no podremos afirmar que dicha interacción sea determinante para la Distancia o la Cercanía.

Esto no nos puede llevar a pensar que nuestros registros son atribuibles a otros contextos y a otras interacciones, todavía es necesario complementar estos trabajos con una muestra mayor e incluso compararlos con los resultados académicos para conocer hasta dónde la interacción afecta el aprendizaje.

Pero, sobre todo, realizar un estudio de cada curso en los Grados. En trabajos anteriores, Palomino, Marchena y Ramos (2017) estudiaron la interacción profesorado-alumnado comparando dos etapas educativas. Concluyeron que la etapa de Secundaria mostraba más Cercanía que Primaria y la edad, por tanto, condiciona la interacción. Esto puede indicarnos que, si las edades influyen, las interacciones del alumnado de primer curso de Grado y de cuarto curso deben ser por tanto diferentes.

\section{Bibliografía}

Ainscow, M. (200I). Necesidades especiales en el aula. Guía para la formación del profesorado. Madrid: Narcea/unEsCo.

Alonso Tapia, J. (2005). Motivación para el aprendizaje: la perspectiva de los alumnos. En La orientación escolar en centros educativos (pp. 209-242). Madrid: MEC.

Bahillo, G.; Puyal, E.; SAnz, A.; Elboj, C. y San Agustín, M. (2006). Las relaciones de convivencia y conflicto escolar en los centros educativos aragoneses de enseñanza no universitaria. Zaragoza: Gobierno de Aragón.

Benito, M. (2009). Debates en torno a la enseñanza de las ciencias. Revista Perfiles Educativos, $3 I(123), 27-43$.

Bigss (2005) citado por Aguilar González, K. A. (20I4). Factores personales y contextuales relacionados con el clima social en el aula en educación media superior (Doctoral dissertation, Universidad Autónoma de Nuevo León).

Briones, S. (200I). Las tecnologías de la información y la comunicación: Su impacto en la educación. Pixel-Bit: Revista de Medios y Educación, 17, 67-789. 
Cabaní, M. y Carretero, M. (2003). La promoción de los estudiantes estratégicos a través del proceso de evaluación que proponen los profesores universitarios. En C. Monereo i FonT y J. I. Pozo Municio (eds.), La universidad ante la nueva cultura educativa en la universidad: Enseñar y aprender para la autonomía (pp. 173-190). Madrid: Síntesis.

Carbonero, M. Á.; Martín Antón, L. J. y Reoyo, N. (2OII). El profesor estratégico como favorecedor del clima de aula. European Journal of Education and Psychology, 4(2), diciembre, I33-I42.

Carrasco, C. y Trianes, M. V. (20Io). Clima social, prosocialidad y violencia como predictores de inadaptación escolar en primaria. European Journal of Education and Psychology, 3(2), 229-242.

Coll, C. (2003). El currículo universitario en el siglo xxi. En C. Monereo i Font y J. I. Pozo Municio (eds.) La universidad ante la nueva cultura educativa en la universidad: Enseñar $y$ aprender para la autonomía (pp. 27I-284). Madrid: Síntesis.

Coronel, J. M. (1994). El profesorado, el liderazgo y la mejora de la escuela. Qurriculum: Revista de teoría, investigación y práctica educativa, 8(9), 2I-48.

Depaepe, M. (2006). Vieja y nueva historia de la educación: Ensayos críticos. Barcelona: Octaedro.

Doyle, W. (1979). Classroom tasks and students' abilities. En P. L. Peterson y H. J. Walberg (eds.), Research on teaching: Concepts, findings, and implications (pp. 183-209). Berkeley, CA: McCutchan.

Echeverri, G. y López, B. (2005). El currículo universitario: una propuesta compleja. Ponencia: I Seminario Nacional. Universidad de la Amazonia, octubre 5-7. Florencia.

Escudero, J. M. (coord.) (1997). Diseño y Desarrollo del currículum en la Educación Secundaria. Barcelona: ICE-Horsori.

Evertson, C. M. y Green, J. L. (1989). La observación como indagación y método. En M. C. Wiтtrock (coord.) La investigación de la Enseñanza, II. Métodos cualitativos y de observación (pp. 303-370). Barcelona: Paidós.

Fabra, M. y Doménech, M. (200I). Hablar y Escuchar. Relatos de profesores y estudiantes. Barcelona: Paidós.

FERnÁndez García, J. A. (2008). Modelo explicativo del rendimiento en secundaria: variables cognitivas del profesor y cognitivas, afectivas, motivacionales y escolares de los alumnos. Departamento de Psicología. Universidad de Valladolid. Tesis doctoral.

Fernández Pérez, M. (1989). Así enseña nuestra universidad. Salamanca: Hispagraphis.

Gairín, J. (1999). La organización Escolar: Contexto y texto de actuación. Madrid: Muralla.

Gairín, J. (2003) El profesor universitario en el siglo xxi. En C. Monereo y J. I. Pozo (eds.) La universidad ante la nueva cultura educativa. Enseñar y aprender para la autonomía. Madrid: Síntesis.

Garbanzo, G. (2007). Factores asociados al rendimiento académico. Revista Educación de la Universidad de Costa Rica, 3I(I), 43-63.

Glaser, B. y Strauss, A. (1967). The Discovery of Grounded Theory: Strategies for Qualitative Research. New York: Aldine Publishing Company.

Goodson, I. (I99I). La construcción social del currículum. Posibilidades y ámbitos de investigación de la historia del currículum. Revista de Educación, 295, 7-30.

Goodson, I. (2000). El cambio en el currículum. Barcelona: Octaedro.

Hannan, A. y Siver, H. (2005). La innovación en la enseñanza superior: Enseñanza, aprendizaje y culturas institucionales. Madrid: Narcea.

Hattie, J. y Clinton, J. (200I). The assessment of teachers. Teaching Education, I2(3), 279-297. doi:I0.1080/1047621012009655I.

Heras Montoya, L. (1997) Comprender el espacio educativo: investigación etnográfica sobre un centro escolar. Información General. Archidona (Málaga): Aljibe. ISBN: 84-87767-60-5.

Hernández, A. (2005). Los factores afectivos y el aprendizaje significativo en la docencia universitaria. Tesis de Maestría no publicada. Universidad Nacional (CIDE), Maestría en Docencia Universitaria. Heredia, Costa Rica. 
IвÁÑ̃E, N. (2002). Las emociones en el aula. Estudios pedagógicos, 28, 31-45.

Illera (2003). Tecnologías y aprendizajes en la universidad. En C. Monereo y J. Pozo (eds.), La universidad ante la nueva cultura educativa: Enseñar y aprender para la autonomía (pp. 26I-270). Madrid: Síntesis.

López, C. y Morante, B. (2004). Estudio de casos. En F. Salvador Mata, J. L. Rodríguez Diéguez y A. Bolívar Botia, Diccionario enciclopédico de didáctica. Málaga: Aljibe.

Marchena, R. (2005). Mejorar el ambiente de las clases de Secundaria: un enfoque práctico para responder a la diversidad desde el aula. Málaga: Editorial Aljibe.

Marchena, R. (2007). La interacción alumnado/profesorado, clave para la mejora del clima de aprendizaje en el aula. En J. Moreno y F. Luengo (coords. Proyecto Atlántida). Construir ciudadanía y prevenir conflictos: la elaboración de planes de convivencia en los centros. Madrid: Wolters Kluwer España, S. A.

Marchena, R.; Palomino, A.; Ramos, E. y Montesdeoca, R. (20I7). Las clases de educación física: ¿qué dicen los profesores y qué hacen los alumnos? desde una perspectiva etnográfica. Revista Acción Motriz, I8, 7-I8.

Martín, P. A. (2007). Evaluación formativa y su repercusión en el clima del aula. Revista de Investigación Educativa, 25(2), 389-402.

Merchán, J. (2005). Crítica de la Didáctica: conocimiento, formación del profesorado y mejora de la enseñanza. Revista de Investigación en la Escuela, 55, 29-40.

Merchán, J. (2009). La cuestión del cambio de la práctica de la enseñanza y la necesidad de una teoría de la acción en el aula. Revista Iberoamericana de Educación, 48(6), I-II.

Milicic, N. y AArón, A. (2004). Clima social escolar y desarrollo personal. Santiago de Chile: Andrés Bello.

Molina de Colmenares, N. y Pérez de Maldonado, I. (2006). El clima de relaciones interpersonales en el aula un caso de estudio. Revista Paradigma (Maracay), 37(2), 193-219.

Monereo, C. y Pozo, J. (2003). La cultura educativa en la universidad: nuevos retos para profesores y alumnos. En C. Monereo y J. Pozo (eds.), La universidad ante la nueva cultura educativa: Enseñar y aprender para la autonomia (pp. 15-32). Madrid: Síntesis.

Mora, E. y Pujal i Llombart, M. (20I7). La tensión entre el cuidado y el servicio en la didáctica universitaria. Revista Iberoamericana de Educación Superior, 8(22), 45-63.

Palomino, A.; Marchena, R. y Ramos, E. (2017). Educación física en primaria y secundaria: Análisis comparativo de la interacción profesorado-alumnado. Profesorado. Revista de Curriculum y Formación de Profesorado, 2I(2), 253-270.

Pereira, Z. (20Io). Las dinámicas interactivas en el ámbito universitario: el clima de aula. Revista Electrónica Educare, I4(58), 7-20.

Pérez Serrano, G. (1994). Investigación cualitativa. Retos e interrogantes I y H. Madrid: La Muralla.

Perrenoud, P. (2005). Diez nuevas competencias para enseñar. Revista Educatio Siglo XXI, 23, 223-229.

Real Decreto 1393/2007, de 29 de octubre, por el que se establece la ordenación de las enseñanzas universitarias oficiales. Ministerio de Educación y Ciencia. BOE 260, 30 de octubre de 2007.

Ríos, D. (2010). Factores que inciden en el clima de aula universitario. Revista Latinoamericana de Estudios Educativos. México, D.F., 40(3), I05-I26.

Saballs, T. y Castañer, J. C. (2002). Formación del profesorado orientada al desarrollo de competencias de gestión del aula de Eso; el afrontameinto de situaciones críticas. [Documento en línea]. Disponible en Rev. Elect. Interuniv. Form. Profr., 5(I), I575-0965.

SABUCEDo, A. C. (200I). Observación y análisis de los procesos de aula en la universidad: una perspectiva holística. Enseñanza E Teaching: Revista Interuniversitaria de Didáctica, I9, I8I-208.

Santos Rego, M. Á. (2017). La calidad del profesorado en el futuro de la universidad. El desafío de la formación pedagógica. Edetania: Estudios y Propuestas Socioeducativas, 52, 197-217. Serrano, G. P. (1994). Investigación cualitativa: retos e interrogantes. Métodos. La Muralla. 
STAKE, R. E. (2005). Qualitative case studies. Madrid: Morata.

TAPIA, J. (2005). Motivar en la escuela, motivar en la familia: claves para el aprendizaje. Madrid: Morata.

TikunoffF, W. J.; Ward, B. A. y Griffin, G. A. (1979). Interactive research and development on teaching study: Final report. San Francisco: Far West Laboratory for Educational Research and Development.

Tomlimson, A. (200I). El aula diversificada. Barcelona: Octaedro.

Valle, A.; CaAbanach, R. y Rodríguez, S. (2006). Reflecting on motivation and learning in the new Spanish education act (LOE). Talking vs doing. Revista Papeles del PsicóLogo, 27(3), I35-I38.

VAlle Olmos, M. D. (2013). La comunicación como herramienta para mejorar el clima y el proceso de aprendizaje en las aulas universitarias: diagnóstico y propuestas. Valladolid. Trabajo Fin de Máster. Recuperado de http://uvadoc.uva.es/handle/I0324/378o.

Voli, F. (2004). Sentirse bien en el aula: Manual de convivencia para profesores. Madrid: Educar.

Walker, R. (1983). La realización de estudios de casos en educación. Ética, teoría y procedimientos. En W. Dockrell y D. Hamilton (eds.). Nuevas reflexiones sobre la investigación educativa (pp. 42-82). Madrid: Narcea.

YIN, R. (1989). Case study research: Design and methods, revised edition. California: Cosmos Corporation. 